OPEN

SUBJECT AREAS:

GROWTH FACTOR

SIGNALLING

REPROGRAMMING

Received

27 October 2014

Accepted

18 November 2014

Published

4 December 2014

Correspondence and requests for materials should be addressed to P.D. (dping@koto.kpum.ac.jp) or T.T. (ttakam@koto.kpu-m. ac.jp)

\section{Combining TGF- $\beta$ signal inhibition and connexin43 silencing for iPSC induction from mouse cardiomyocytes}

Ping Dai' ', Yoshinori Harada' ', Hitoshi Miyachi ${ }^{2}$, Hideo Tanaka', Satsuki Kitano ${ }^{2}$, Tetsuya Adachi' Tomoyuki Suzuki ${ }^{1}$, Hitoshi Hino' \& Tetsuro Takamatsu'

'Department of Pathology and Cell Regulation, Graduate School of Medical Science, Kyoto Prefectural University of Medicine, 465 Kawaramachi-Hirokoji, Kamigyo-ku, Kyoto 602-8566, Japan, ${ }^{2}$ Experimental Research Center for Infectious Diseases, Institute for Virus Research, Kyoto University, 53 Shogoin, Kawara-cho, Sakyo-ku, Kyoto 606-8507, Japan.

The reprogramming of differentiated cells into induced pluripotent stem cells (iPSCs) can be achieved by ectopic expression of defined transcription factors (Oct3/4, Sox2, Klf4 and c-Myc). However, to date, some iPSCs have been generated using viral vectors; thus, unexpected insertional mutagenesis in the target cells would be a potential risk. Here we report reprogramming of siPSCs (gene silencing-induced pluripotent stem cells) from mouse neonatal cardiomyocytes (CMs) by combining TGF- $\beta$ signal inhibition and connexin43 (Cx43) silencing, and show that siPSCs show pluripotency in vitro and in vivo. Our novel non-insertional mutagenesis technique may provide a means for iPSC generation.

T he generation of iPSCs from somatic cells has been achieved by the overexpression of four transcriptionfactor-encoding genes, i.e., Oct3/4, Klf4, Sox2, and c-Myc, in the mouse ${ }^{1}$, $\mathrm{rat}^{2,3}, \mathrm{pig}^{4}$, and monkey ${ }^{5}$, and in humans ${ }^{6,7}$. The original method of iPSC generation uses viral vectors, such as retroviruses and lentiviruses, which integrate the reprogramming factor into the host genome ${ }^{1}$. Particularly, one of the factors, $\mathrm{c}-\mathrm{Myc}$, is a known proto-oncogene, and its excess activation could give rise to transgene-derived tumour formation ${ }^{8}$. To mitigate the risk of genomic insertions of exogenous reprogramming factors for iPSC generation, non-viral and non-integrating viral methods have been developed and produced iPSCs with potentially reduced risks ${ }^{9-18}$. However, most of the methods developed still involve the exogenous ectopic expression of defined transcription factors; thus the potential risk for unexpected genetic modifications by the exogenous sequences in the target cells is not completely avoidable.

Inhibition of TGF- $\beta$ signaling in conjunction with the defined transcription factors significantly improves the efficiency of iPSC generation from mouse and human fibroblasts ${ }^{19-22}$. Previously, we and others have shown that TGF- $\beta$ signaling is positively mediated by $\mathrm{Cx} 43^{23,24}$, a major gap-junctional protein, which is strongly expressed in $\mathrm{CMs}^{23}$. In addition, $\mathrm{Cx} 43$ is considered to be a typical embryonic stem (ES) cell marker ${ }^{25}$, and also has a major function in the regulation of self-renewal and maintenance of pluripotency in ES cells ${ }^{26}$. Consequently, this evidence led us to reason that combining TGF- $\beta$ signal inhibition and $\mathrm{Cx} 43$ silencing would serve as a novel method for generating transgene-free iPSCs, and would have a direct impact on the reprogramming of mouse CMs.

\section{Results}

We first tested the TGF- $\beta$ receptor I (T $\beta$ RI)/ALK5 inhibitor SB- 431542 and Cx43 small interference RNA (Cx43 siRNA $)^{27}$ on mouse neonatal primary CMs for their effect on reprogramming. The mouse neonatal primary CMs express abundant endogenous $\mathrm{Cx} 43$ (Supplementary Fig. 1). We confirmed that naked FITC-labeled Cx43 siRNA penetrated into CMs (Supplementary Fig. 2), and that the endogenous $\mathrm{Cx} 43$ protein of CMs was significantly reduced by $\mathrm{Cx} 43$ siRNA (Supplementary Fig. 1 and 3). To determine the reprogramming capability of SB- 431542 or $\mathrm{Cx} 43$ siRNA, on day 7 after culture, the CMs were switched from primary CM Dulbecco's modified Eagle's medium (DMEM) to normal mouse embryonic stem cell (mESC) growth media containing either SB-431542 $(10 \mu \mathrm{M}), \mathrm{Cx} 43$ siRNA (100 nM) or both compounds (Fig. 1a), and were maintained over the next 2-4 weeks. Two weeks after treatment, we obtained two siPSC colonies per $2 \times 10^{5}$ cells, yielding an overall reprogramming efficiency of $\sim 0.001 \%$, when the cells were treated with a combination of SB-431542 and Cx43 siRNA 
$\mathbf{a}$

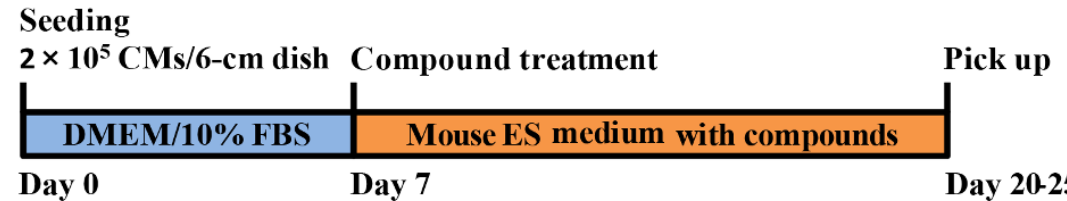

b
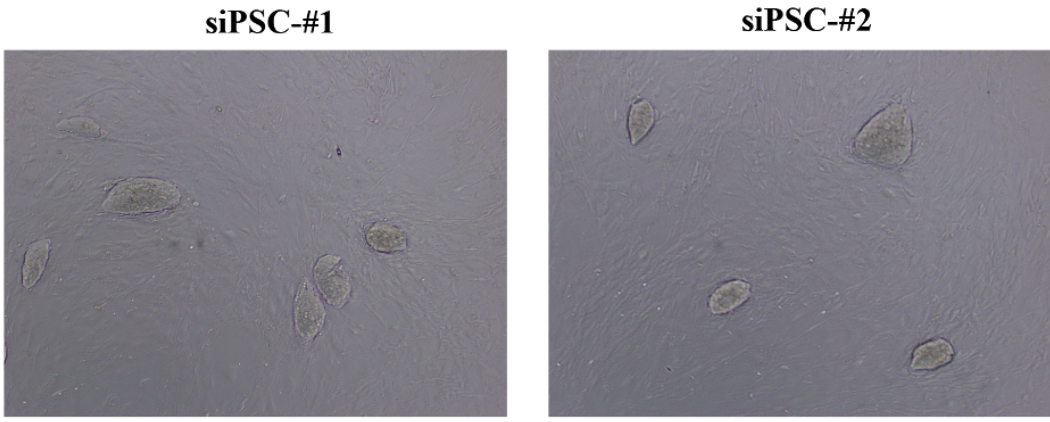

c

siPSC-\#1

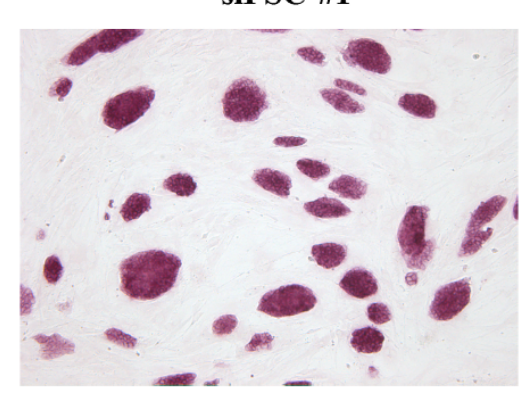

siPSC-\#2

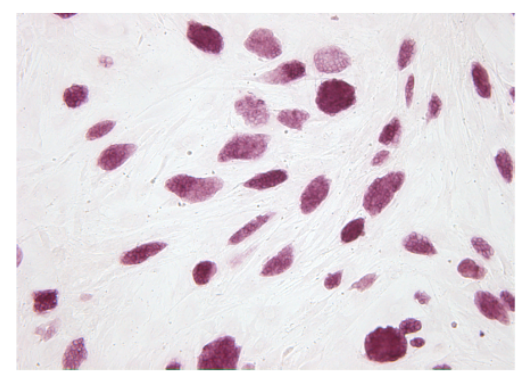

Figure 1 Generation of siPSCs from mouse CMs by the combination of TGF- $\beta$ signal inhibition and Cx43 silencing. (a) Protocol for mouse siPSC generation by combining inhibition of TGF- $\beta$ signal and Cx43 silencing. (b) Morphology of established siPSCs. Phase-contrast images of siPSC colonies are shown. siPSC-\#1 and -\#2 refer to independent siPSC colonies. (c) siPSCs stained for ALP, a typical pluripotency marker.

(Supplementary Table 1). The two generated siPSC colonies were expanded for over 40 passages stably and homogeneously and showed characteristic mESC-like morphology (Fig. 1b). These colonies were positive for alkaline phosphatase (ALP) (Fig. 1c), an early pluripotency marker, suggesting that they might be reprogrammed cells. The initial siPSCs were subsequently passaged under mESC growth conditions to yield siPSCs for further characterisation. In contrast, up to 4 weeks, none of these types of colonies were observed in cultures treated with SB-431542 or Cx43 siRNA alone (Supplementary Table 1), or in cultures treated with a nonsense control siRNA alone or combined SB-431542 and the control siRNA (data not shown). Moreover, we obtained an overall reprogramming efficiency of $\sim 0.0015 \%$ siPSC colonies ( $\alpha$ MHC-siPSC) per $2 \times 10^{5}$ cultured $\mathrm{CMs}$ isolated from hearts derived from $\alpha \mathrm{MHC}$-Cre mice after crossbreeding with mice carrying floxed genetic markers, when the cells were treated with a combination of SB431542 and $\mathrm{Cx} 43$ siRNA in three independent experiments (Supplementary Table 2). However, up to 4 weeks, none of these types of colonies were observed in cultured CMs treated with both SB-431542 $(10 \mu \mathrm{M})$ and Cx40 siRNA $(100 \mathrm{nM})$, which was used as a negative control of $\mathrm{Cx} 43$ siRNA, and/or gap junction protein inhibitor, Octanol $(500 \mu \mathrm{M})$ or Doxyl stearic acid (DSA) $(50 \mu \mathrm{M})$ (Supplementary Table 2). In addition, we also obtained three colonies from $2 \times 10^{5}$ cultured CMs of C57BL/6-Tg (CAG-EGFP) mice (Supplementary Fig. 4a).

The generated murine siPSC colonies expressed typical pluripotency embryonic markers, including Nanog, Oct4, Sox 2 and SSEA1 (Fig. 2a). Reverse transcription-polymerase chain reaction (RTPCR) and quantitative real-time PCR analysis confirmed that these siPSC colonies had a high level of endogenous gene expression respectively (Fig. 3, with Supplementary Table 3 and Fig. 2b-f, with Supplementary Table 4). Similar results were also obtained in $\alpha$ MHC-siPSC colonies (Supplementary Fig. 5 and Supplementary Table 4). Bisulfite genomic sequencing analyses of the Nanog and Oct4 proximal promoters showed that both were as hypomethylated in siPSCs as in mESCs, while they were hypermethylated in CMs (Fig. 4a, b). They showed a normal karyotype (Supplementary Fig. 6).

In the examination of the pluripotency of siPSC in vitro differentiation through the formation of embryoid bodies (EBs), siPSCs were found to efficiently form EBs in suspension (Fig. 5a) and EBs could be differentiated into cell types of multiple derivatives, including ectodermal cells characterised by staining of typical neuron marker $\beta$ III Tubulin (Tuj1) (Fig. 5b), mesodermal cells revealed as physiologically functional differentiated CMs (Fig. 5c and Supplementary Videos 1 and 2), and endothelial cells stained positively for CD31 (Fig. 5d). Furthermore, RT-PCR analysis of the two siPSC colonies derived from CMs confirmed the typical marker gene expression of all three embryonic germ layers (Fig. 6 and Supplementary Table 3).

To examine the pluripotency of siPSCs in vivo, we subcutaneously injected each of the two siPSC colonies into dorsal flanks of severe combined immunodeficient (SCID) mice. Five weeks after injection, we observed teratoma formation. Histological examination revealed that the two siPSC colonies differentiated into all three germ layers, including neural tissues (ectoderm) (Fig. 7a) and epidermal tissue (ectoderm) (Fig. 7b), cartilage (mesoderm) (Fig. 7c), and gland (endoderm) (Fig. 7d). Furthermore, we tested whether siPSCs contributed to the formation of chimeric mice when injected into postcompacted eight-cell ICR mouse embryos. The siPSC colonies contributed $20-60 \%$ to chimera formation, as judged by coat colour (Fig. 7e and Supplementary Fig. 4b). Finally, we used the most highly 


\section{$\mathbf{a}$}
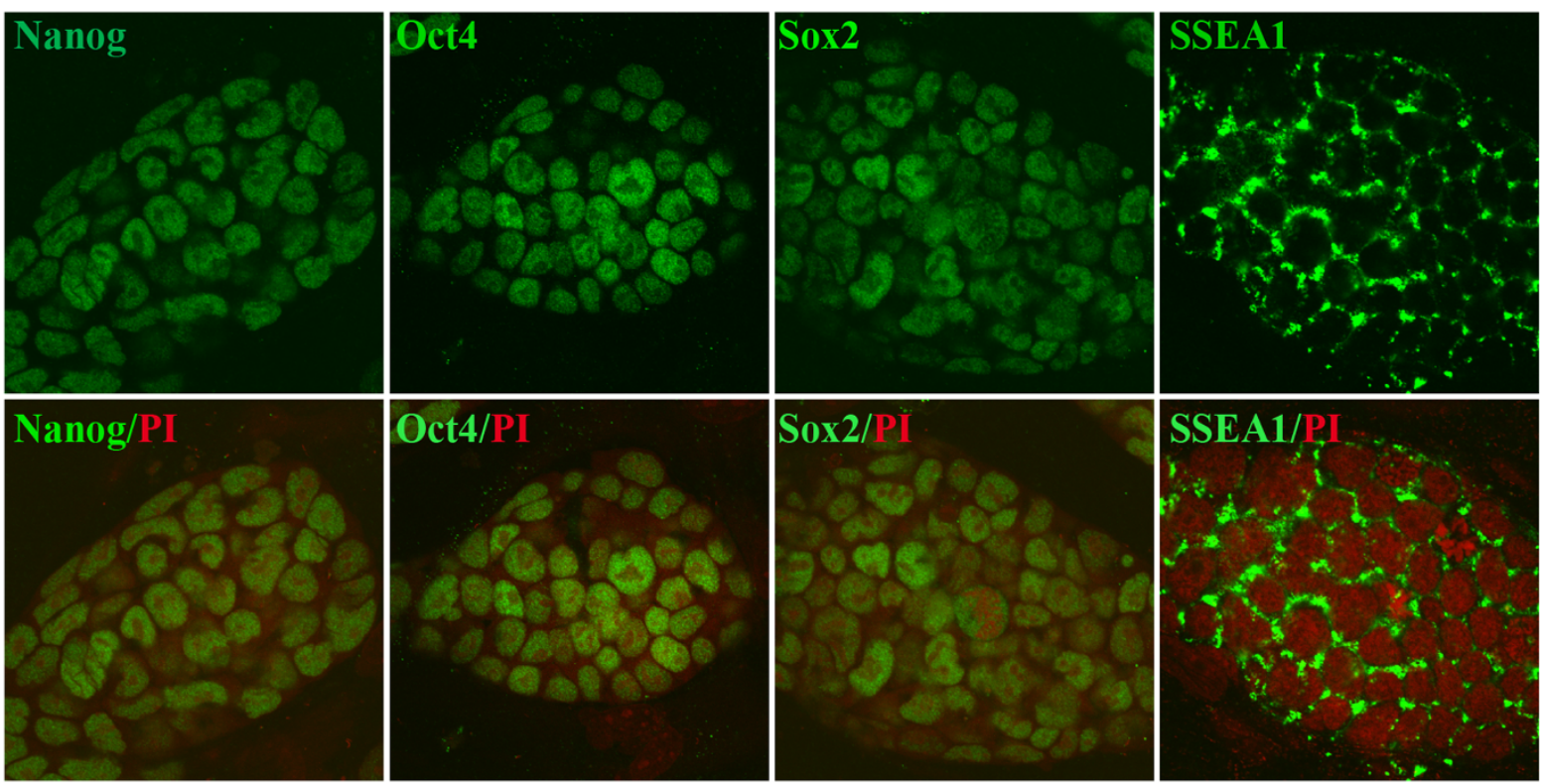

b

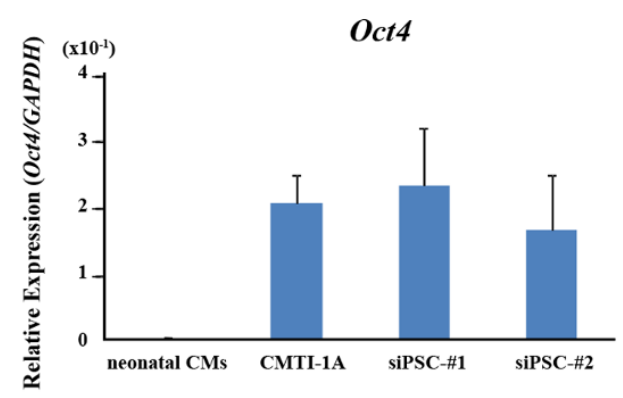

C
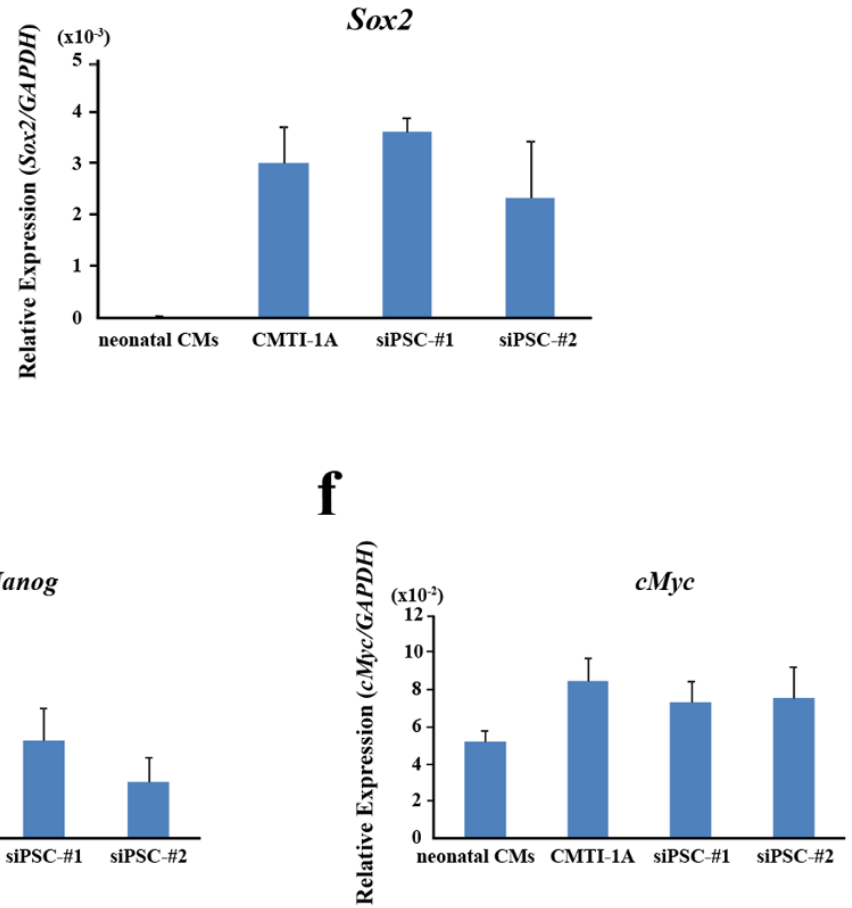

Figure $2 \mid$ Characterisation of reprogrammed siPSC colonies. (a) Immunostaining images of siPSCs show protein expression of the typical pluripotency markers Nanog, Oct4, Sox2 and SSEA1. (b-f) Quantitative real-time PCR analysis of cDNA from two siPSC colonies. mRNA expression of Oct4 (b), Sox2 (c), Klf4 (d), Nanog (e) and $c M y c$ (f) was normalised against the control gene, GAPDH, expression.

contributive chimera to test germline transmission by crossing it with ICR mice. The results confirmed germline transmission (Fig. 7f).

\section{Discussion}

These in vitro and in vivo characterisations collectively confirm that the TGF- $\beta$ pathway inhibition and transient silencing of $\mathrm{Cx} 43$ by siRNA are sufficient to reprogram CMs to become siPSCs, which are morphologically and functionally similar to conventional mESCs
(Fig. 1 and Supplementary Fig. 4, and Supplementary Tables 1 and 2 ). Regarding the potential mechanism of gene silencing reprogramming, it is reported that TGF- $\beta$ signaling could replace either Sox 2 or c-Myc in iPS cell generation from $\mathrm{MEF}^{21}$. $\mathrm{Cx} 43$ down-regulation in ES cells affects such measures as Oct3/4 and Nanog expression ${ }^{26}$. In addition, the addition of leukemia inhibitory factor (LIF, contained in ES medium) is important for the self-renewal of iPS cells in the mouse and human ${ }^{6,8}$, and activates the expression of Klf4, which was noted as one of the downstream targets of LIF/Stats signaling in 


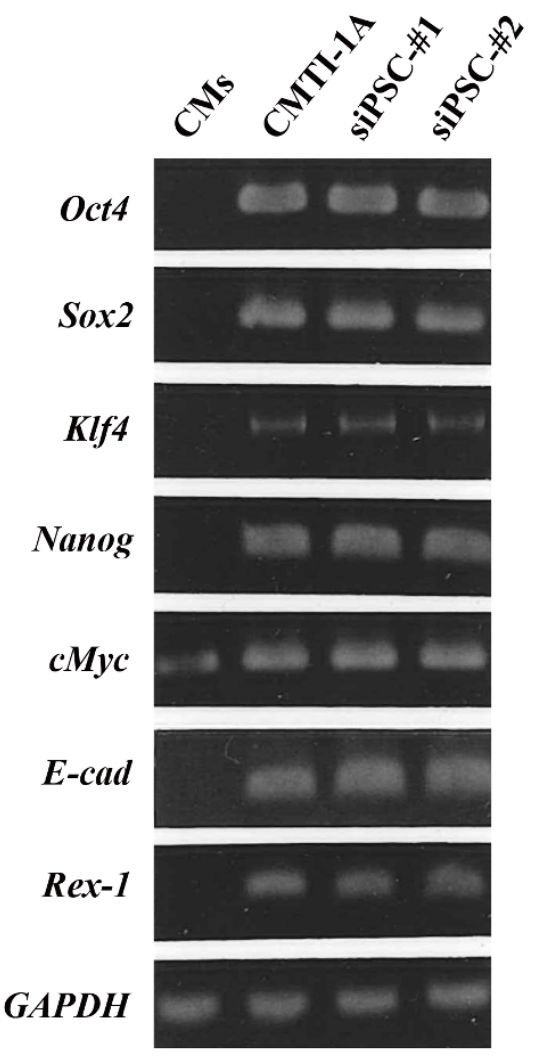

Figure 3 RT-PCR analysis of cDNA from two siPSC colonies. CMTI-1A, an mESC line. Endogenous pluripotency gene expression of Oct4, Sox2, Klf4, Nanog, $c M y c, E$-cad and Rex-1, and the expression of control gene GAPDH were analysed.

mouse ES cells ${ }^{28}$. Therefore inhibition of TGF- $\beta$ signaling and Cx43 silencing could foster the reprogramming process in multiple ways. Here, we describe a novel method for generating transgene-free iPS cells, and show that siPSCs from mouse neonatal primary CMs generated by the combination of TGF- $\beta$ signal inhibition and $\mathrm{Cx} 43$ silencing are self-renewing and pluripotent in vitro and in vivo. Therefore, combining inhibition of TGF- $\beta$ signaling and transient silencing of Cx43 by siRNA or other methods may be useful in generating integration-free iPS cells for future medical applications.

\section{Methods}

Primary CM isolation and culture. Primary CMs were isolated from 2-day-old neonatal C57BL/6 or C57BL/6-Tg (CAG-EGFP) mouse hearts (Shimizu Laboratory Supplies Corp., Ltd.) and/or derivative mouse hearts derived from $\alpha$ MHC-Cre, i.e., B6. Cg-Tg (Myh6-cre/Esr1*)1Jmk/J mice ${ }^{29}$ (Jackson Laboratories, Bar Harbor, ME, USA) after crossbreeding with B6. Cg-Tg (CAG-floxed Neo-EGFP) REP080sb mice ${ }^{30}$ provided by the REKEN BRC through the National Bio-Resource Project of the MEXT, Japan. Briefly, ventricles were removed under sterile conditions from neonatal mice, placed in cold sterile calcium-free phosphate buffered saline (PBS), minced into approximately 2 -mm cubes, and treated with $1 \mathrm{mg} \mathrm{ml}^{-1}$ collagenase (type II; Worthington Biochemical Corp.). Dissociated cells were preplated for 45 min in DMEM (Sigma) containing 10\% heat-inactivated fetal bovine serum (FBS). After the incubation, the suspension, containing mostly CMs, was collected. This process was repeated four times. The resulting CMs were resuspended in DMEM containing $10 \% \mathrm{FBS}, 100 \mathrm{U} \mathrm{ml}^{-1}$ penicillin, and $100 \mathrm{gg} \mathrm{m}^{-1}$ streptomycin, and isolated CMs from hearts derived from $\alpha \mathrm{MHC}$-Cre mice after crossbreeding with mice carrying floxed genetic markers were resuspended in DMEM containing 10\% FBS, $100 \mathrm{U} \mathrm{ml}^{-1}$ penicillin, $100 \mu \mathrm{g} \mathrm{ml}^{-1}$ streptomycin, and $400 \mu \mathrm{g} \mathrm{ml}^{-1} \mathrm{G} 418$, and seeded in standard culture dishes for reprogramming of siPSCs. All animal experiments were conducted with the approval of and in accordance with guidelines from the Committee for Animal Research, Kyoto Prefectural University of Medicine.

Reprogramming of siPSCs. CMs were seeded at $2 \times 10^{5}$ cells in a 6 -cm dish coated with $0.1 \%$ gelatin (Sigma) in DMEM supplemented with $10 \%$ FBS. On day 7 , the CMs were switched from DMEM medium to normal mESC growth media (GIBCO) supplemented with $100 \mathrm{U} \mathrm{ml}^{-1}$ leukemia inhibitory factor (LIF, Millipore) containing either SB-431542 (10 $\mu \mathrm{M})$ (Tocris), Cx43 siRNA (sense: $5^{\prime}$-CAA UUC UUC UUG CCG CAA TT- $3^{\prime}$; antisense: $5^{\prime}$-UUG CGG CAA GAA GAA UUG TT-3') $(100 \mathrm{nM})$ or both compounds, and as controls, the medium containing either SB$431542(10 \mu \mathrm{M})$, nonsense control siRNA (sense: 5'-AAU UCU CCG AAC GUG UCA CGT-3'; antisense: 5' -GUG ACA CGU UCG GAG AAU UTT-3') (100 nM) or both compounds, and/or both SB-431542 $(10 \mu \mathrm{M})$ and Cx40 siRNA (Santa Cruz, sc43079) (100 nM), which was used as a negative control of Cx43 siRNA. To confirm the effect of gap junction protein inhibitors on reprogramming of siPSCs from CMs, the medium contained both SB-431542 $(10 \mu \mathrm{M})$ and Octanol (Sigma-Aldrich) $(500 \mu \mathrm{M})$ or Doxyl stearic acid (DSA) (Sigma-Aldrich) $(50 \mu \mathrm{M})$. To confirm penetration of naked Cx43 siRNA into CMs, we used a FITC-labeled Cx43 siRNA into CMS. The media were changed every 3 days. Colonies with morphologies similar to $\mathrm{mESC}$ colonies were clearly visible by day 17 after compound treatment. At days 20-25 after compound treatment, siPSC colonies were individually picked up for further expansion and analysis.

Cytochemistry and immunofluorescence assay. ALP staining was performed using the Alkaline Phosphatase Detection Kit (Chemicon) as instructed by the manufacturer. Immunocytochemistry was performed using standard protocol. Briefly, cells were fixed in $2 \%$ paraformaldehyde (Sigma-Aldrich), washed three times with PBS, and then incubated in PBS containing $0.1 \%$ Triton X-100 and 3\% skim milk in PBS for $1 \mathrm{~h}$ at room temperature. The cells were then incubated with the following a

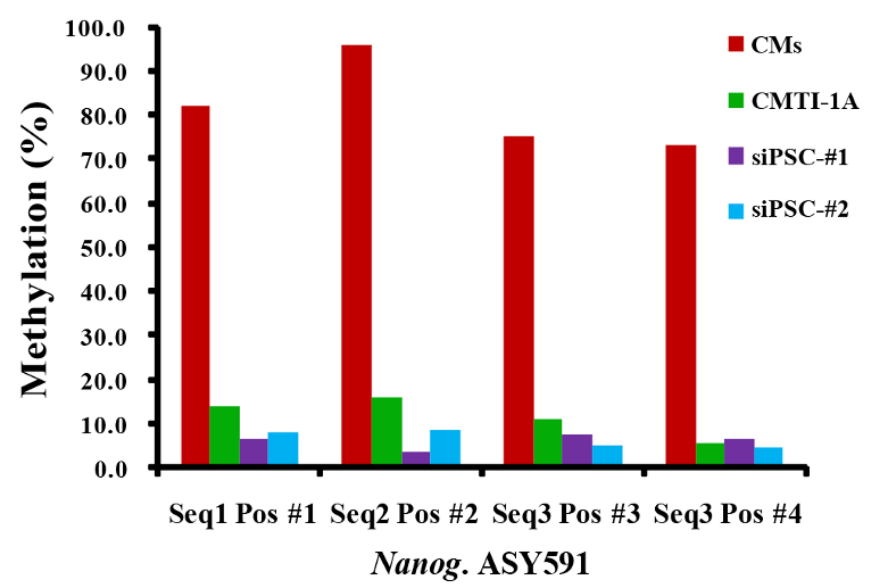

b

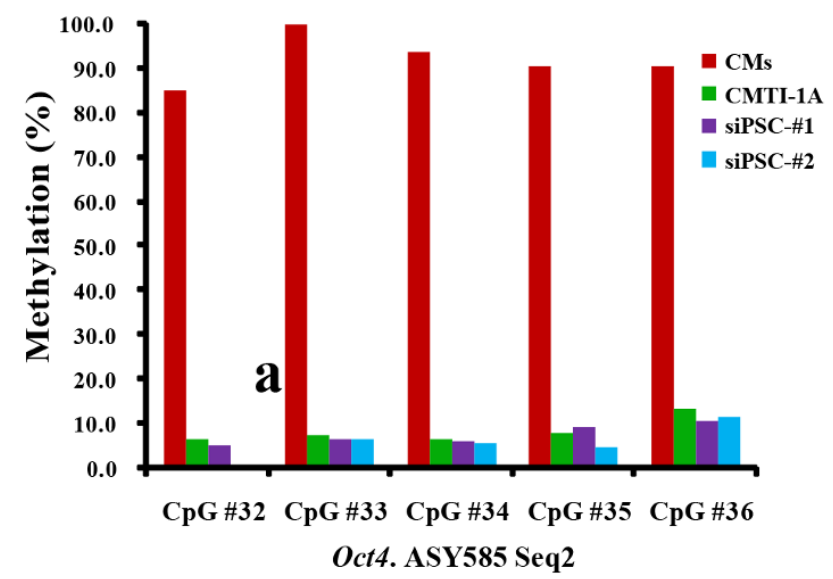

Figure 4 | Bisulfite genomic sequencing analyses of the Nanog and Oct4 proximal promoters. (a) Bisulfite pyrosequencing measuring methylation in the proximal promoter regions of Nanog in the indicated cells. (b) Bisulfite pyrosequencing measuring methylation in the proximal promoter regions of Oct4 in the indicated cells. 
a

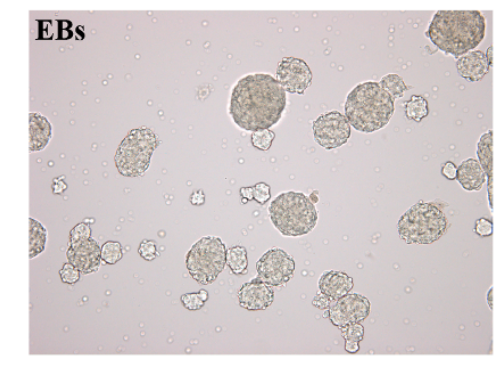

c

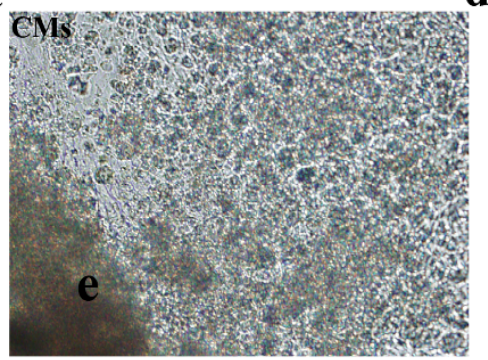

b
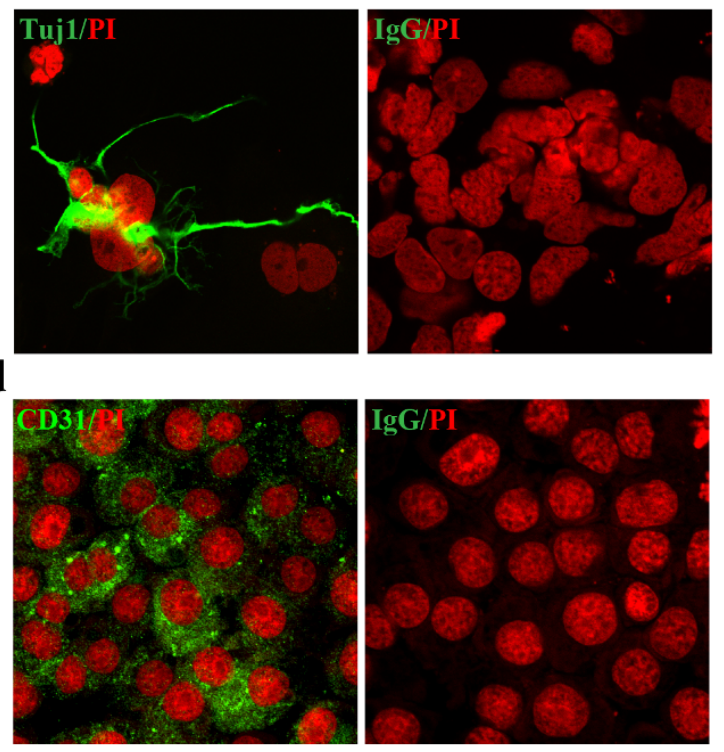

Figure 5 Pluripotency characterisation of siPSCs in vitro. (a) Phase-contrast image of EBs generated from siPSCs. (b) Immunostaining image of siPSCderived ectodermal cells, characterised by immunoreactivity to typical neuron marker $\beta$ III Tubulin (Tuj1) or normal mouse IgG. (c) Phase-contrast image of siPSC-derived mesodermal cells, revealed as differentiated CMs. (d) Immunostaining image of siPSC-derived endodermal cells, stained positively for CD31 or IgG.

primary antibodies at $4{ }^{\circ} \mathrm{C}$ overnight: Nanog (ab21603, 1:500, Abcam); Oct4 (sc5279, 1: 100, Santa Cruz); Sox2 (AB5603, 1:500, Millipore); SSEA1 (sc-21702, 1: 100, Santa Cruz); Tuj-1 (MMS-435P, 1:500, Covance); CD31 (sc-1506-R, 1:100, Santa Cruz); and IgG (sc-2025, 1:500, Santa Cruz). After washing three times with PBS, cells were incubated with secondary antibodies: Alexa Fluor 488 goat anti-mouse IgG ( $1: 1000$, Molecular Probes) and Alexa Fluor 488 goat anti-rabbit IgG ( $1: 1000$, Molecular Probes) for $2 \mathrm{~h}$ at room temperature. Nuclei were detected by propidium iodide (PI) (Vector Laboratories, Inc.) staining. Images were analysed by confocal microscopy (FV1000; Olympus).

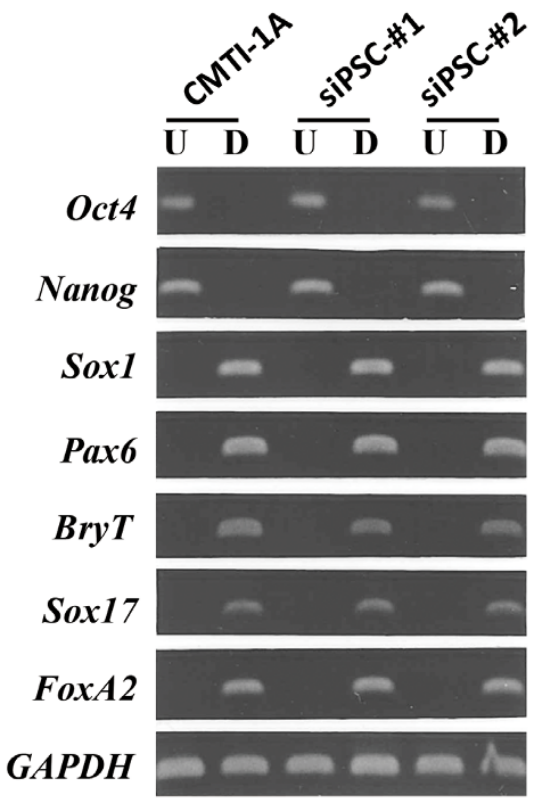

Figure 6 RT-PCR analysis of undifferentiated (U) and differentiated (D) siPSCs for pluripotency markers. Pluripotency markers (Oct4 and Nanog) and various differentiation markers for three germ layers, including ectoderm (Sox1 and Pax6), mesoderm (Brachyury, BryT) and endoderm (Sox17 and FoxA2).
RNA preparation and RT-PCR analysis. Total RNA was prepared using RNeasy Mini Kit (Qiagen), and cDNA synthesized according to product instructions using SuperScriptTM II First-Strand Synthesis kit (Invitrogen) for RT-PCR. PCRs were carried out with Taq DNA polymerase (Qiagen). PCR products were resolved on 1.5\% agarose gels and visualised by ethidium bromide staining. All primer sequences are listed in Supplementary Table 2. For quantitative real-time PCR, the LightCycler TaqMan Master Kit was used for cDNA amplification of specific target genes. The expression of mRNA was normalized against control gene, GAPDH, expression. All primer sequences for real-time PCR analysis are listed in Supplementary Table 4.

Bisulfite pyrosequencing. We used a service provider (EpigenDx),

In vitro differentiation. The pluripotency of siPSCs was examined by in vitro differentiation from EBs. To form EBs, siPSCs were trypsinised into single cells and cultured in suspension on low-adhesion plates (Corning) in mESC medium containing $15 \%$ knockout serum (Invitrogen), $4.5 \mathrm{~g} \mathrm{~L}^{-1} \mathrm{~L}$-glutamine, $1 \%$ nonessential amino acids, $0.1 \mathrm{mM} 2$-mercaptoethanol, 50 units ml $^{-1}$ penicillin, $50 \mu \mathrm{g} \mathrm{ml}^{-1}$ streptomycin, and $100 \mathrm{U} \mathrm{ml}^{-1}$ LIF. Media were refreshed every 3 days, and EBs were allowed to grow for 8 days in suspension and then replated onto a $0.1 \%$ gelatin-coated tissue culture dish. Spontaneous differentiations of siPSCs into cells of mesodermal, endodermal, and ectodermal lineages were then detected by RT-PCR (primers are listed in Supplementary Table 2) and with appropriate markers by immunofluorescence. Directed differentiation of siPSCs to neurons, CMs, and endothelial cells followed previously published protocols ${ }^{6,31}$.

Calcium imaging of siPSC-derived CMs. Clusters of differentiated CMs derived from siPSCs were incubated in $10-\mathrm{cm}$ culture dishes and loaded with fluo-4/AM $\left(0.625 \mathrm{mg} \mathrm{ml}^{-1}\right)$ for $15 \mathrm{~min}$ at $37^{\circ} \mathrm{C}$, and were subsequently incubated with fluo-4free Tyrode's solution for $15 \mathrm{~min}$ as previously described ${ }^{32}$. Calcium images of the cells were obtained from the fluo- 4 fluorescence intensities by using a confocal system composed of an upright microscope with a spinning-disc confocal scanner (CSU-21; Yokogawa) under excitation with an argon laser at 100 frames s$^{-1}$. The fluorescence image data were stored at 100 frames s $^{-1}$ into the computer via a MiCaM-02 imaging system (Brainvision) ${ }^{33}$.

Teratoma formation. To examine the in vivo developmental potential of mouse siPSCs, we injected $5 \times 10^{5}$ cells from each of the two donor iPS cell lines into individual mice, and repeated the experiment a total of three times ( $\mathrm{n}=6$ mice total). After five weeks, teratomas from all mice were dissected and fixed in $4 \%$ paraformaldehyde. Samples were embedded in paraffin and processed with haematoxyline and eosine staining or antibody staining with Tuj-1 (PRB-435P, $1: 2000$, Covance). All procedures were performed in accordance with guidelines from the Committee for Animal Research, Kyoto Prefectural University of Medicine.

Chimera formation and germline transmission. siPSCs were injected into denuded eight-cell stage embryos flushed from 2.5 days post-coitum (dpc) ICR female mice, and transferred into the uteruses of $2.5 \mathrm{dpc}$ ICR pseudopregnant recipient females. Chimerism was ascertained after birth by the appearance of coat colour (from siPSCs) 

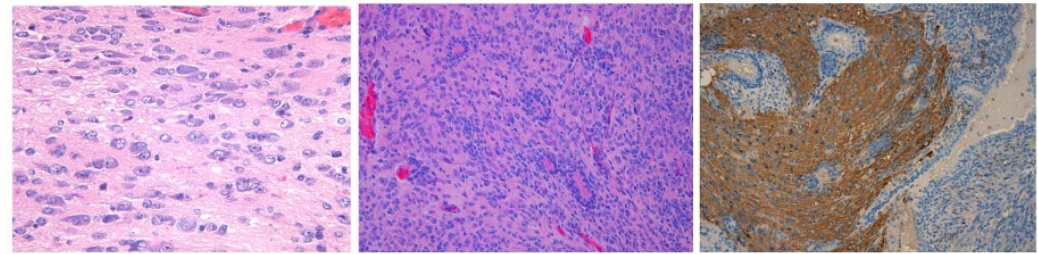

b

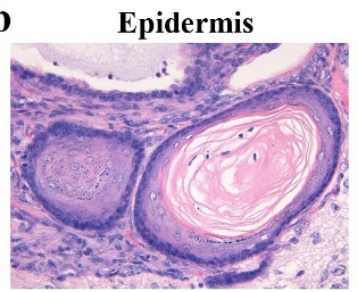

e

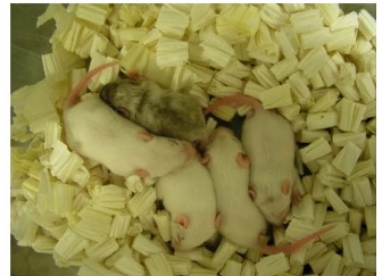

c

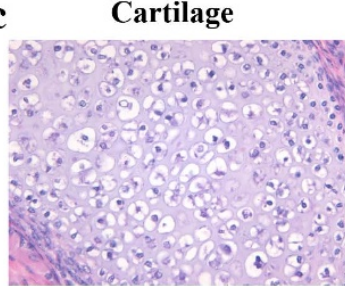

d

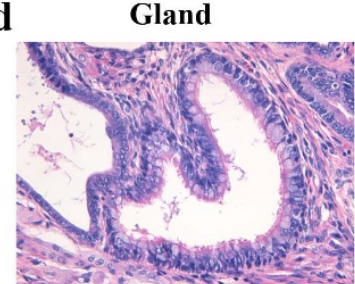

f

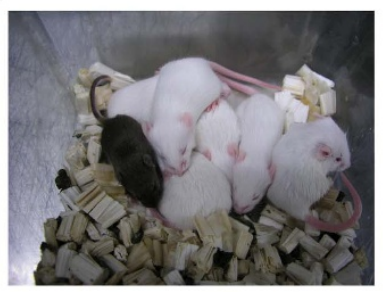

Figure $7 \mid$ Pluripotency characterisation of siPSCs in vitro. (a-d) Subcutaneous injection of siPSCs caused teratomas to develop in SCID mice. Representative histological sections of teratomas show that they consist of all three embryonic germ layers. (a) Neural tissues (ectoderm) (left panel), neural rosettes (middle panel), and immunoreactivity to typical neuron marker $\beta I I I$ Tubulin (Tuj1) (right panel). (b) Epidermis (ectoderm), (c) cartilage (mesoderm), and (d) gland (endoderm). (e) Chimeric mouse derived from siPSCs. (f) Coat colour of offspring shows germline transmission.

in white host pups. High-contribution chimeras were mated to ICR mice to test for germline transmission.

1. Takahashi, K. \& Yamanaka, S. Induction of pluripotent stem cells from mouse embryonic and adult fibroblast cultures by defined factors. Cell 126, 663-676 (2006).

2. Li, W. et al. Generation of rat and human induced pluripotent stem cells by combining genetic reprogramming and chemical inhibitors. Cell Stem Cell 4, 16-19 (2009).

3. Liao, J. et al. Generation of induced pluripotent stem cell lines from adult rat cells. Cell Stem Cell 4, 11-15 (2009).

4. Esteban, M. A. et al. Generation of induced pluripotent stem cell lines from Tibetan miniature pig. J. Biol. Chem. 284, 17634-17640 (2009).

5. Liu, H. et al. Generation of induced pluripotent stem cells from adult rhesus monkey fibroblasts. Cell Stem Cell 3, 587-590 (2008).

6. Takahashi, K. et al. Induction of pluripotent stem cells from adult human fibroblasts by defined factors. Cell 131, 861-872 (2007).

7. Yu, J. et al. Induced pluripotent stem cell lines derived from human somatic cells. Science 318, 1917-1920 (2007).

8. Okita, K., Ichisaka, T. \& Yamanaka S. Generation of germline-competent induced pluripotent stem cells. Nature 448, 313-317 (2007).

9. Yu, J. et al. Human induced pluripotent stem cells free of vector and transgene sequences. Science 324, 797-801 (2009).

10. Stadtfeld, M., Nagaya, M., Utikal, J., Weir, G. \& Hochedlinger, K. Induced pluripotent stem cells generated without viral integration. Science 322, 945-949 (2008).

11. Okita, K., Nakagawa, M., Hyenjong, H., Ichisaka, T. \& Yamanaka, S. Generation of mouse induced pluripotent stem cells without viral vectors. Science 322, 949-953 (2008).

12. Kaji, K., Norrby, K., Paca, A., Mileikovsky, M., Mohseni, P. \& Woltjen, K. Virusfree induction of pluripotency and subsequent excision of reprogramming factors. Nature 458, 771-775 (2009).

13. Woltjen, K. et al. piggyBac transposition reprograms fibroblasts to induced pluripotent stem cells. Nature 458, 766-770 (2009)

14. Yusa, K., Rad, R., Takeda, J. \& Bradley, A. Generation of transgene-free induced pluripotent mouse stem cells by the piggyBac transposon. Nat. Methods 6, 363-369 (2009).

15. Seki, T. et al. Generation of induced pluripotent stem cells from human terminally differentiated circulating T cells. Cell Stem Cell 7, 11-14 (2010).

16. Zhou, H. et al. Generation of induced pluripotent stem cells using recombinant proteins. Cell Stem Cell 4, 381-384 (2009).
17. Kim, D. et al. Generation of human induced pluripotent stem cells by direct delivery of reprogramming proteins. Cell Stem Cell 4, 472-476 (2009).

18. Warren, L. et al. Highly efficient reprogramming to pluripotency and directed differentiation of human cells with synthetic modified mRNA. Cell Stem Cell 7, 618-630 (2010)

19. Ichida, J. K. et al. A small-molecule inhibitor of tgf-Beta signaling replaces sox 2 in reprogramming by inducing nanog. Cell Stem Cell 5, 491-503 (2009).

20. Lin, T. et al. A chemical platform for improved induction of human iPSCs. Nat Methods 6, 805-808 (2009).

21. Maherali, N. \& Hochedlinger, K. Tgfbeta signal inhibition cooperates in the induction of iPSCs and replaces Sox 2 and cMyc. Curr. Biol. 19, 1718-1723 (2009).

22. Zhu, S. et al. Reprogramming of human primary somatic cells by OCT4 and chemical compounds. Cell Stem Cell 7, 651-655 (2010).

23. Dai, P., Nakagami, T., Tanaka, H., Hitomi, T. \& Takamatsu, T. Cx43 mediates TGF-beta signaling through competitive Smads binding to microtubules. Mol. Biol. Cell 18, 2264-2273 (2007).

24. Asazuma-Nakamura, Y., Dai, P., Harada, Y., Jiang, Y., Hamaoka, K. \& Takamats T. Cx43 contributes to TGF-beta signaling to regulate differentiation of cardiac fibroblasts into myofibroblasts. Exp. Cell Res. 315, 1190-1199 (2009).

25. Aasen, T. et al. Efficient and rapid generation of induced pluripotent stem cells from human keratinocytes. Nat. Biotechnol. 26, 1276-1284 (2008).

26. Todorova, M. G., Soria, B. \& Quesada, I. Gap junctional intercellular communication is required to maintain embryonic stem cells in a nondifferentiated and proliferative state. J. Cell Physiol. 214, 354-362 (2008).

27. Nakano, Y., Oyamada, M., Dai, P., Nakagami, T., Kinoshita, S. \& Takamatsu, T. Connexin 43 knockdown accelerates wound healing but inhibits mesenchymal transition after corneal endothelial injury in vivo. Invest. Ophthalmol. Vis. Sci. 49, 93-104 (2008).

28. Li, Y., McClintick, J., Zhong, L., Edenberg, H. J., Yoder, M. C. \& Chan, R. J. Murine embryonic stem cell differentiation is promoted by SOCS-3 and inhibited by the zinc finger transcription factor Klf4. Blood 105, 635-637 (2005).

29. Sohal, D. S. et al. Temporally regulated and tissue-specific gene manipulations in the adult and embryonic heart using a tamoxifen-inducible Cre protein. Cir. Res. 89, 20-25 (2001).

30. Matsumura, H., Hasuwa, H., Inoue, N., Ikawa, M. \& Okabe, M. Lineage-specific cell disruption in living mice by Cre-mediated expression of diphtheria toxin A chain. Biochem. Biophys. Res. Commun. 321, 275-279 (2004).

31. Okada, Y., Shimazaki, T., Sobue, G. \& Okano, H. Retinoic-acid-concentrationdependent acquisition of neural cell identity during in vitro differentiation of mouse embryonic stem cells. Dev. Biol. 275, 124-142 (2004).

32. Nakagami, T. et al. Generation of reentrant arrhythmias by dominant-negative inhibition of connexin 43 in rat cultured myocyte monolayers. Cardiovasc. Res. 79 70-79 (2008). 
33. Fujiwara, K., Tanaka, H., Mani, H., Nakagami, T. \& Takamatsu, T. Burst emergence of intracellular $\mathrm{Ca} 2+$ waves evokes arrhythmogenic oscillatory depolarization via the $\mathrm{Na}+-\mathrm{Ca} 2+$ exchanger: simultaneous confocal recording of membrane potential and intracellular $\mathrm{Ca} 2+$ in the heart. Cir. Res. 103, 509-518 (2008).

\section{Acknowledgments}

We thank S. Ishii and T. Shinagawa (RIKEN Tsukuba Institute) for discussion.

\section{Author contributions}

P.D. and T.T. designed the experiments and wrote the manuscript; P.D. conducted most of the experiments in this study; Y.H. performed teratoma experiments; H.M. and S.K. performed chimera formation and germline transmission studies; H.T. performed the calcium imaging of differentiated CMs; A.T. performed real-time PCR analysis; T.S. and H.H. isolated CMs; all authors read and approved the manuscript.

\section{Additional information}

Supplementary information accompanies this paper at http://www.nature.com/ scientificreports

Competing financial interests: The authors declare no competing financial interests.

How to cite this article: Dai, P. et al. Combining TGF- $\beta$ signal inhibition and connexin 43 silencing for iPSC induction from mouse cardiomyocytes. Sci. Rep. 4, 7323; DOI:10.1038/ srep07323 (2014).

(c) (i) (2) This work is licensed under a Creative Commons Attribution-NonCommercialShareAlike 4.0 International License. The images or other third party material in this article are included in the article's Creative Commons license, unless indicated otherwise in the credit line; if the material is not included under the Creative Commons license, users will need to obtain permission from the license holder in order to reproduce the material. To view a copy of this license, visit http:// creativecommons.org/licenses/by-nc-sa/4.0/ 